\title{
Calogero Model(s) and Deformed Oscillators
}

\author{
Marijan MILEKOVIĆ ${ }^{\dagger}$, Stjepan MELJANAC $\ddagger$ and Andjelo SAMSAROV \\ ${ }^{\dagger}$ Physics Department, Faculty of Science, Bijenička c. 32, 10002 Zagreb, Croatia \\ E-mail: marijan@phy.hr \\ ¥ Rudjer Bošković Institute, Bijenička c. 54, 10002 Zagreb, Croatia \\ E-mail: meljanac@irb.hr
}

Received November 30, 2005, in final form March 02, 2006; Published online March 17, 2006

Original article is available at http://www.emis.de/journals/SIGMA/2006/Paper035/

\begin{abstract}
We briefly review some recent results concerning algebraical (oscillator) aspects of the $N$-body single-species and multispecies Calogero models in one dimension. We show how these models emerge from the matrix generalization of the harmonic oscillator Hamiltonian. We make some comments on the solvability of these models.
\end{abstract}

Key words: Calogero model; deformed oscillator algebra; $S_{N}$-extended Heisenberg algebra

2000 Mathematics Subject Classification: 81R12; 81R15; 81Q05; 46L65

\section{Introduction}

There exists a very limited number of exactly solvable many-body systems, even in one dimension (1D) [1, 2] and the Calogero model [3, 4] is surely one of the most famous and exhaustively studied examples of such models. In its original version, the Calogero model describes $N$ identical, spinless, nonrelativistic particles on the line which interact through an inverse-square two-body interaction and are subjected to a common confining harmonic force. Although being $1 \mathrm{D}$ problem, its solution is far from being straightforward and it is really amazing how this model and its various descedants, known as Calogero-Sutherland-Moser systems [5], have so deep and profound impact on various branches of physics and mathematics, ranging from condensed matter systems and black hole physics $[6,7]$ to random matrices and strings [8,9]. Why is this so and what motivates physicist and mathematicians to study such a class of models? Maybe the best answer, more that three decades ago, was offered by Calogero himself in the opening section of his seminal paper [4]. In his own words "A motivation is perceived in the insight that exact solutions, even of oversimplified models, may provide and in the possibility to assess the reliability of approximation techniques that can be used in more realistic contexts, by first testing them in exactly solvable cases. Moreover, for some physical problems a 1-dimensional schematization may indeed be appropriate."

The single-species $N$-body Calogero model is defined by the following Hamiltonian $(i, j=$ $1,2, \ldots, N)$ :

$$
H=-\frac{\hbar^{2}}{2 m} \sum_{i} \frac{\partial^{2}}{\partial x_{i}^{2}}+\frac{m \omega^{2}}{2} \sum_{i} x_{i}^{2}+\frac{\hbar^{2} \nu(\nu-1)}{2 m} \sum_{i \neq j} \frac{1}{\left(x_{i}-x_{j}\right)^{2}},
$$

where $\nu(\nu-1) \equiv g$ is the dimensionless coupling constant, equal for all particles. The wellknown stability condition requires $g \geq-1 / 4$. The constant $m$ is the mass of particles and $\omega$ is the strength of a common harmonic confinement potential. The ground state of the Hamiltonian (1) 
is known to be of the highly correlated, Jastrow form

$$
\Psi_{0}\left(x_{1}, x_{2}, \ldots, x_{N}\right)=\left(\prod_{i<j}\left|x_{i}-x_{j}\right|^{\nu}\right) e^{-\frac{m \omega}{2 \hbar} \sum_{i} x_{i}^{2}} \equiv \Delta e^{-\frac{m \omega}{2 \hbar} \sum_{i} x_{i}^{2}} .
$$

The corresponding ground state energy depends on $\nu$ explicitly and is given by

$$
E_{0}=\omega\left(\frac{N}{2}+\frac{\nu N(N-1)}{2}\right) .
$$

As conjectured by Calogero [3], the remainder of the eigenspectrum of the model (1) coincides (except for a constant shift of all energy levels) with the energy spectrum of the system of free harmonic oscillators. An explicit mapping of the model (1) to the set of $N$ free harmonic oscillators was found quite recently in [10] and later applied to the other Calogero-like systems [11].

One can solve this model exactly and find out the complete set of energy eigenvalues either by following the traditional approach $[3,4,12]$ (see also the recent paper by Hallnas and Langmann [13], who briefly review this and some other approaches, including their own new solution algorithm for solving (1)) or by employing its underlying $S_{N}$ (permutational) algebraic structure [14]. The later approach, based on the particular set of creation and annihilation operators, is considerably simpler than the original one [3, 4] and yields an explicit expression for the wavefunctions in terms of action of creation operators on the ground state (vacuum). We review this approach in Section 2.

The single-species Hamiltonian (1) can be easily generalized to the multispecies variant of the model $[15,16]$ and even to the arbitrary dimensions [17]. The multispecies Calogero model, as a generalization of the Calogero model (1) to the system of distinguishable particles, is worth to study for (at least) two reasons. It exhibits some novel features not encountered in the model with identical particles and represents a playground for the testing mathematical tools, developed earlier for the single-species Calogero model (see comments below). It is also related to the notion of the Haldane exclusion statistics [18] and the origin of this relation can be traced back to the model (1). Namely, the Calogero model (1) provides a microscopic realization of the generalized Haldane exclusion statistics, where the role of the Haldane statistical parameter is played by the coupling constant $g$ in the inverse-square interaction [19, 20]. In Haldane's formulation of statistics there is a possibility of having particles of different species, with a statistical parameter depending on the species coupled. Thus, the extension of the model (1) to the multispecies case is a natural step [21, 22, 23]. We also mention that the simplest multispecies model, with just two different kind of particles [16], has been recently reconsidered in the supersymmetric framework and it has been claimed that the corresponding Hamiltonian might describe the motion of electrons in a one-dimensional superconductor [24].

Distinguishability of the species can be introduced by allowing particles to have different masses $\left(m \rightarrow m_{i}\right)$ and different couplings $\left(\nu \rightarrow \nu_{i j}\right)$ to each other. (In fact, this generalization has already been suggested in [4], although not using statistical arguments.) The common feature of these generalizations (including higher dimensional cases) is a new long-range threebody interaction that appears in the Hamiltonian. Its character and strength is determined by the parameters of the two-body interaction. In 1D multispecies variant of (1), this three-body interaction can be switched off by a suitable chosen connection between coupling constants $\nu_{i j}$ and masses $m_{i}$ of the particles but for the models in $D>1$ this interaction is generic and cannot be turned off. Contrary to the 1D single-species model (1), in all above cases only a limited set of exact eigenstates and eigenenergies are known and the complete solution of the multispecies problem, even in $1 \mathrm{D}$, is still lacking. It is interesting that the $S_{N}$-based approach (in a slightly generalized form), so successfully used in [14] to solve Hamiltonian (1), does not hold for the 1D multispecies model. Instead of $S_{N}$ structure, one can try to employ underlying $\mathrm{SU}(1,1)$ 
structure of the multispecies Hamiltonian and define an another set of creation and annihilation operators (construct from $\mathrm{SU}(1,1)$ generators) but, as we explain in Section 4, this approach also has limitations.

Section 3 is an interlude. We show how single- and multispecies 1D Calogero models emerge from the matrix generalization of the single harmonic oscillator Hamiltonian [25], i.e. we adopt a view that the both models represent, in a some sense, a "deformed" harmonic oscillator, "deformation" being encoded in an unusual commutation relations. Section 5 is a concluding section.

\section{Single-species Calogero model in 1D and $S_{N}$-extended Heisenberg algebra}

In this section we briefly review an operator solution to the Calogero model (1), based on $S_{N}$-extended Heisenberg algebra, and discuss Fock space of this algebra. We closely follow references $[14,26,27]$.

In order to simplify the analysis, we extract Jastrow factor $\Delta$ from the ground state $\Psi_{0}\left(x_{1}, x_{2}\right.$, $\left.\ldots, x_{N}\right)$ and define new vacuum $|\tilde{0}\rangle=\tilde{\Psi}_{0}=\Delta^{-1} \Psi_{0}$. This generates a similarity transformation on Hamiltonian (1), which leads to an another Hamiltonian $\tilde{H}$ i.e. $\tilde{H}=\Delta^{-1} H \Delta$.

We find $\tilde{H}$ as

$$
\tilde{H}=-\frac{1}{2 m} \sum_{i=1}^{N} \frac{\partial^{2}}{\partial x_{i}^{2}}+\frac{m \omega^{2}}{2} \sum_{i=1}^{N} x_{i}^{2}-\frac{1}{2 m} \sum_{i \neq j} \frac{\nu}{\left(x_{i}-x_{j}\right)}\left(\frac{\partial}{\partial x_{i}}-\frac{\partial}{\partial x_{j}}\right) .
$$

Instead of the Hamiltonian (1), it is then convenient to work with the transformed Hamiltonian $\tilde{H}$. We define a covariant derivative $D_{i}[14,28]$ by

$$
D_{i}=\partial_{i}+\nu \sum_{j \neq i} \frac{1}{\left(x_{i}-x_{j}\right)}\left(1-K_{i j}\right),
$$

that close under the commutation, $\left[D_{i}, D_{j}\right]=0$. Here $K_{i j}$ 's are the generating elements of the symmetric group $S_{N}$

$$
\begin{array}{ll}
K_{i j}=K_{j i}, \quad\left(K_{i j}\right)^{2}=1, & K_{i j}^{\dagger}=K_{i j}, \\
K_{i j} K_{j l}=K_{j l} K_{i l}=K_{i l} K_{i j}, & i \neq j, \quad i \neq l, \quad j \neq l,
\end{array}
$$

which interchange the particles with labels $i$ and $j$, for example $K_{i j} x_{j}=x_{i} K_{i j}$.

In terms of covariant derivatives and coordinates one can define ladder operators $a_{i}$ and $a_{i}^{\dagger}$ as (we put all unimportant constants equal to one):

$$
a_{i}^{\dagger}=\frac{1}{\sqrt{2}}\left(x_{i}-D_{i}\right), \quad a_{i}=\frac{1}{\sqrt{2}}\left(x_{i}+D_{i}\right) .
$$

The physical space of $N$ identical particles is represented either by symmetric or antisymmetric wave functions. It can be shown that all necessary information is actually contained in the totally symmetric space. Restriction to the totally symmetric space yields Hamiltonian

$$
\tilde{H}=\Delta^{-1} H \Delta=\frac{1}{2} \sum_{i}\left\{a_{i}, a_{i}^{\dagger}\right\}=\sum_{i} a_{i}^{\dagger} a_{i}+E_{0}=\frac{1}{2} \sum_{i}\left(X_{i}^{2}+P_{i}^{2}\right),
$$

where $P_{i}=-\imath D_{i}$ and $X_{i}=x_{i}$. In deriving these expressions, we used the commutation rules that follow directly from the definitions (5) and (6):

$$
\left[X_{i}, P_{i}\right]=\imath \delta_{i j}\left(1+\nu \sum_{k=1}^{N} K_{i k}\right)-\imath \nu K_{i j}
$$




$$
\left[a_{i}, a_{j}^{\dagger}\right]=\delta_{i j}\left(1+\nu \sum_{k=1}^{N} K_{i k}\right)-\nu K_{i j}, \quad\left[a_{i}^{\dagger}, a_{j}^{\dagger}\right]=\left[a_{i}, a_{j}\right]=0
$$

together with the vacuum conditions $a_{i}|\tilde{0}\rangle=0, K_{i j}|\tilde{0}\rangle=+|\tilde{0}\rangle$.

The algebra of creation and annihilation operators (7) is known as $S_{N}$-extended Heisenberg

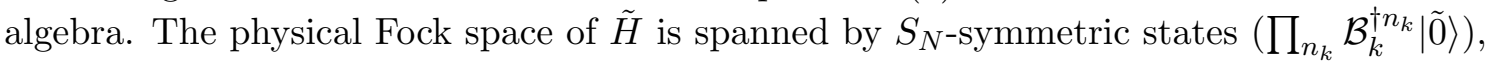

$$
\tilde{H}\left(\prod_{n_{k}} \mathcal{B}_{k}^{\dagger n_{k}}|\tilde{0}\rangle\right)=\left[E_{0}+\sum_{k=1}^{N} k n_{k}\right]\left(\prod_{n_{k}} \mathcal{B}_{k}^{\dagger n_{k}}|\tilde{0}\rangle\right),
$$

where $\mathcal{B}_{k}=\sum_{i} a_{i}^{k}$ are collective, $S_{N}$-symmetric operators. For further details about algebra of these operators and Fock space structure we refer the reader to references [29, 30].

As it stands, the algebra (7) is consistent for all values of the parameter $\nu$. The only additional restriction on the $\nu$ may come from the representation of the algebra on the Fock space. For example, the states in the complete Fock space of algebra (7) should have positive norm. Apart from its particular realization, $S_{N}$-invariant Heisenberg algebra is basically a multimode oscillator algebra with permutational invariance. We developed earlier a general techniques for analyzing such a class of algebras [27, 31]. Information about structure of the Fock space is encoded either in (i) action of annihilation operators on monomial states in Fock space, $a_{i}\left(a_{1}^{\dagger n_{1}} \cdots a_{N}^{\dagger n_{N}}|\tilde{0}\rangle\right)$ or in (ii) Gram matrices of scalar products in Fock space, $A_{i_{N} \cdots i_{1} ; j_{1} \cdots j_{N}}=\left\langle\tilde{0}\left|a_{i_{N}} \cdots a_{i_{1}} a_{j_{1}}^{\dagger} \cdots a_{j_{N}}^{\dagger}\right| \tilde{0}\right\rangle$. For the algebra (7) there is no closed form available for the approach (i) but one can use (slightly cumbersome) recursion relations for it [27], which may help to obtain matrix elements of the Gram matrix (ii).

The Gram matrix for the one-particle states $a_{i}^{\dagger}|\tilde{0}\rangle$ is of order $N$ and has only two distinct entries. The Gram matrix for two-particle states $a_{i}^{\dagger} a_{j}^{\dagger}|\tilde{0}\rangle$ is of order $N^{2}$ and has four distinct entries and so on. For the convenience, we display below the Gram matrix of one-particle states, i.e.

$$
\left(\begin{array}{cccc}
1+\nu(N-1) & -\nu & \cdots & -\nu \\
-\nu & 1+\nu(N-1) & \cdots & -\nu \\
\cdots & \cdots & \cdots & \cdots \\
-\nu & -\nu & \cdots & 1+\nu(N-1)
\end{array}\right)
$$

Its eigenvalues and eigenvectors are

\begin{tabular}{|c|c|c|c|}
\hline Eigenvalue & Degeneracy & Eigenvector & Comments \\
\hline \hline 1 & 1 & $\mathcal{B}_{1}^{\dagger}|\tilde{0}\rangle$ & \\
\hline $1+N \nu$ & $N-1$ & $\left(a_{1}^{\dagger}-a_{i}^{\dagger}\right)|\tilde{0}\rangle$ & $i \neq 1$ \\
\hline \hline
\end{tabular}

This Gram matrix is positive definite, i.e. there are no eigenvectors with negative norm if $\nu>-\frac{1}{N}$. At $\nu=-\frac{1}{N}$, there is a critical point where the $(N-1)$ states $\left(a_{1}^{\dagger}-a_{i}^{\dagger}\right)|0\rangle$ have zero norm. One can show that for two-, three-, and many-particle states the same condition for positivity of eigenvalues is required [26, 27]. There exists the universal critical point at $\nu=-\frac{1}{N}$, where all matrix elements of the arbitrary $k$-multi-state Gram matrix are equal to $\frac{k !}{N^{k}}$. In the limit $\nu N \rightarrow-1$ the system of deformed oscillators (7) exhibits singular behaviour. There survives only one oscillator, of the type $\mathcal{B}_{1}^{\dagger}$, describing the motion of the center-of-mass coordinate. All other $(N-1)$ relative coordinates collapse into the same point-the center of mass. This means that the relative coordinates, the relative momenta and the relative energy are all zero at the critical value of the parameter $\nu$. This can be also verified independently by applying large- $N$ collective field theory to the Hamiltonian $\tilde{H}$ [26]. However, one should bear in 
mind that the interval $\nu \in\left(-\frac{1}{N}, 0\right)$ is physically acceptable for the Hamiltonian $\tilde{H}$, describing $N$ oscillators with $S_{N}$-extended Heisenberg algebra, but it is not allowed for the original Calogero Hamiltonian (1) since the wave functions, containing Jastrow factor, diverge at the coincidence point for negative values of $\nu$. This reminds the fact that the two Hamiltonians are not unitary (i.e. physically) equivalent.

\section{Interlude: matrix quantum deformed oscillators and emergence of the Calogero-like models}

In the preceding section we have seen that the Hamiltonian $\tilde{H}$ was still given as quadratic form in coordinates $X_{i}=x_{i}$ and (generalized) momenta $P_{i}=-\imath D_{i}$, i.e. in the form of the harmonic oscillator Hamiltonian. It is then tempting to start with the most general harmonic oscillator Hamiltonian and try to reproduce Calogero-like Hamiltonian(s). In this section we present, following [25], such construction. The final goal is to obtain, by adjusting parameters, not only a single-species Calogero model (1) but also a multispecies one.

Let us consider a matrix generalization of harmonic oscillator Hamiltonian $(\omega=1)$

$$
\boldsymbol{H}=\frac{1}{2}\left(\boldsymbol{P} M^{-1} \boldsymbol{P}+\boldsymbol{X} M \boldsymbol{X}\right) .
$$

where $M_{i j}=m_{i} \delta_{i j}$ is non-singular mass matrix $\left(m_{i}>0\right)$ and $\boldsymbol{P}=-\imath \boldsymbol{D}$ and $\boldsymbol{X}$ are generalized momentum and coordinate matrices respectively, with operator-valued matrix elements:

$$
\boldsymbol{P}=\left(\begin{array}{cccc}
P_{11} & P_{12} & \cdots & P_{1 N} \\
P_{21} & P_{22} & \cdots & P_{2 N} \\
\cdots & \cdots & \cdots & \cdots \\
P_{N 1} & P_{N 2} & \cdots & P_{N N}
\end{array}\right), \quad \boldsymbol{X}=\left(\begin{array}{cccc}
x_{1} & 0 & \cdots & 0 \\
0 & x_{2} & \cdots & 0 \\
\cdots & \cdots & \cdots & \cdots \\
0 & 0 & \cdots & x_{N}
\end{array}\right)
$$

We assume the following deformed matrix commutation relations:

$$
[\boldsymbol{X}, \boldsymbol{P}]=i \boldsymbol{\Lambda} \quad \Rightarrow \quad[\boldsymbol{X}, \boldsymbol{D}]=-\boldsymbol{\Lambda}
$$

where $\boldsymbol{\Lambda}$ is a Hermitian matrix with constant, real and symmetric matrix elements

$$
\boldsymbol{\Lambda}=\left(\begin{array}{cccc}
1 & 0 & \cdots & 0 \\
0 & 1 & \cdots & 0 \\
\cdots & \cdots & \cdots & \cdots \\
0 & 0 & \cdots & 1
\end{array}\right)+\left(\begin{array}{cccc}
0 & \nu_{12} & \cdots & \nu_{1 N} \\
\nu_{12} & 0 & \cdots & \nu_{2 N} \\
\cdots & \cdots & \cdots & \cdots \\
\nu_{1 N} & \nu_{2 N} & \cdots & 0
\end{array}\right)
$$

In terms of matrix elements, the commutation relations (9) read

$$
D_{i j} x_{j}-x_{i} D_{i j}=\nu_{i j}, \quad D_{i i} x_{i}-x_{i} D_{i i}=1 .
$$

There are many solutions of these equations, since adding a coordinate, i.e. $\boldsymbol{X}$-dependent, part to $\boldsymbol{D}$ does not affect them. We can always transform $\boldsymbol{D}$ by an arbitrary function $F(\boldsymbol{X})$, i.e. $\boldsymbol{D} \rightarrow F(\boldsymbol{X})^{-1} \boldsymbol{D} F(\boldsymbol{X})$. On the level of Hamiltonian this generates a non-unitary transformation $\boldsymbol{H} \rightarrow F(\boldsymbol{X})^{-1} \boldsymbol{H} F(\boldsymbol{X})$.

We restrict ourselves to transformations defined by

$$
F(\boldsymbol{X})=\prod_{i<j}\left(x_{i}-x_{j}\right)^{\theta_{i j}}, \quad \theta_{i j}=\theta_{j i}
$$


A corresponding class of solutions of equations (10) is given by

$$
D_{i j}=\delta_{i j}\left(\frac{\partial}{\partial x_{i}}+\sum_{k \neq i} \theta_{i k} \frac{1}{\left(x_{i}-x_{k}\right)}\right)-\nu_{i j} \frac{\left(1-\delta_{i j}\right)}{\left(x_{i}-x_{j}\right)} .
$$

To summarize: we ended up with a class of (matrix) Hamiltonians that depends on three parameters, namely masses $m_{i}$, "deformation" parameters $\nu_{i j}=\nu_{j i}$ and "gauge" parameters $\theta_{i j}=\theta_{j i}$. To obtain a one-dimensional models from (8), one has to perform a suitable "reduction". The following "reduction" was proposed in [25]:

$$
H=\operatorname{Tr}(\boldsymbol{H} \cdot \boldsymbol{J}),
$$

where the constant matrix $\boldsymbol{J}$ has the form

$$
\boldsymbol{J}=\left(\begin{array}{cccc}
1 & 1 & \cdots & 1 \\
1 & 1 & \cdots & 1 \\
\cdots & \ldots & \ldots & \ldots \\
1 & 1 & \ldots & 1
\end{array}\right)
$$

The "reduction" procedure outlined here differs from that followed in [32] in the way how Calogero model appears. The Hamiltonian obtained here is not just the trace of the matrix Hamiltonian $\boldsymbol{H}$, as in [32], but is given by the trace over $\boldsymbol{H} \cdot \boldsymbol{J}$.

One can easy convince himself that the prescription (11) and the following set of parameters $\left\{m_{i}=m ; \nu_{i j}=\nu ; \theta_{i j}=0\right\}$ leads to Calogero Hamiltonian (1). Similarly, by choosing $\left\{m_{i}=\right.$ $\left.m ; \nu_{i j}=\nu ; \theta_{i j}=\nu_{i j}=\nu\right\}$ one reproduces Hamiltonian (4).

More interesting choice is $\left\{m_{i} ; \nu_{i j}=\nu_{j i} ; \theta_{i j}=0\right\}$, which results in Hamiltonian of multispecies 1D Calogero model, with three-body interaction [15]:

$$
\begin{aligned}
H= & -\frac{1}{2} \sum_{i} \frac{1}{m_{i}} \frac{\partial^{2}}{\partial x_{i}^{2}}+\frac{1}{2} \sum_{i} m_{i} x_{i}^{2}+\frac{1}{4} \sum_{i \neq j} \frac{\nu_{i j}\left(\nu_{i j}-1\right)}{\left(x_{i}-x_{j}\right)^{2}}\left(\frac{1}{m_{i}}+\frac{1}{m_{j}}\right) \\
& +\underbrace{\frac{1}{2} \sum_{i, j, k \neq}\left(\frac{\nu_{i j} \nu_{j k}}{m_{j}}\right) \frac{1}{\left(x_{j}-x_{i}\right)\left(x_{j}-x_{k}\right)}}_{3 \text {-body interaction }} .
\end{aligned}
$$

The multispecies analogue of Hamiltonian $\tilde{H}(4)$, i.e. "gauge" transformed Hamiltonian $\tilde{\boldsymbol{H}}=$ $F(\boldsymbol{X})^{-1} \boldsymbol{H} F(\boldsymbol{X})$, is reproduced by choosing $\left\{m_{i} ; \nu_{i j}=\nu_{j i} ; \theta_{i j}=\nu_{i j}\right\}$, and reads

$$
\tilde{H}=-\frac{1}{2} \sum_{i} \frac{1}{m_{i}} \frac{\partial^{2}}{\partial x_{i}^{2}}+\frac{1}{2} \sum_{i} m_{i} x_{i}^{2}-\frac{1}{2} \sum_{i \neq j} \frac{\nu_{i j}}{\left(x_{i}-x_{j}\right)}\left(\frac{1}{m_{i}} \frac{\partial}{\partial x_{i}}-\frac{1}{m_{j}} \frac{\partial}{\partial x_{j}}\right) .
$$

A few additional remarks concerning the Hamiltonian (12) are in order.

First, it describes distinguishable particles on the line, interacting with harmonic, two-body and three-body potentials. Its ground state reads

$$
\begin{aligned}
& |0\rangle \equiv \Psi_{0}\left(x_{1}, x_{2}, \ldots, x_{N}\right)=\prod_{i<j}\left|x_{i}-x_{j}\right|^{\nu_{i j}} e^{-\frac{1}{2} \sum_{i} m_{i} x_{i}^{2}}=\Delta e^{-\frac{1}{2} \sum_{i} m_{i} x_{i}^{2}}, \\
& E_{0}=\frac{N}{2}+\frac{1}{2} \sum_{i \neq j} \nu_{i j} .
\end{aligned}
$$

and tends to the ground state of the single-species Calogero model (2), (3) in the limit $\nu_{i j} \rightarrow \nu$, $m_{i} \rightarrow m$. The appearance of the generalized Jastrow factor $\Delta=\prod_{i<j}\left|x_{i}-x_{j}\right|^{\nu_{i j}}$ in (12) has 
the same origin as in the Calogero model (1). Namely, because of the singular nature of the Hamiltonian for $x_{i}=x_{j}$, the wave function ought to have a prefactor that vanish for the coincident particles. Also, the stability condition demands that the two-body couplings $g_{i j}=$ $\nu_{i j}\left(\nu_{i j}-1\right)$ should be greater than $-\frac{1}{4}$.

Let us consider the last term in (12) i.e. the three-body interaction, more closely. If we put $m_{j}=m=$ const in (12), symmetrize under the cyclic exchange of the indices $(i \rightarrow j \rightarrow k \rightarrow i)$ and reduce the sum to a common denominator using the identity

$$
\sum_{\text {cycl }} \frac{1}{\left(x_{i}-x_{j}\right)\left(x_{i}-x_{k}\right)}=0
$$

we obtain that the necessary condition for vanishing of the three-body interaction is $\nu_{i j}=\nu$. In this way, the problem (13) is reduced to the ordinary $N$-body Calogero model with harmonic and two-body interactions only.

For the general $\nu_{i j}$ and $m_{j}$, it can be shown that the following conditions for the absence of the three-body term must hold [15]:

$$
\frac{\nu_{i j} \nu_{j k}}{m_{j}}=\frac{\nu_{j k} \nu_{k i}}{m_{k}}=\frac{\nu_{k i} \nu_{i j}}{m_{i}} \quad \forall(i, j, k)
$$

The unique solution of these conditions is $\nu_{i j}=\lambda m_{i} m_{j}, \lambda$ being some universal constant. It should be mentioned that the above arguments cannot be applied in higher dimensions, so the three-body interaction is generic for the higher-dimensional multispecies model $[17,33]$ and makes corresponding Hamiltonian hard to solve.

We note in passing that this particular connection between masses and interaction parameters was also displayed in [22, 34]. In [22], the above conditions arose from the requirement that the asymptotic Bethe ansatz should be applicable to the ground state of a multispecies many-body quantum system obeying mutual (exclusion) statistics, while in [34] its origin was not obvious. We offer the simplest possible interpretation, namely these conditions eliminate the three-body interaction from the Hamiltonian.

\section{Multispecies Calogero model in 1D, $S_{N^{-}}$-extended Heisenberg algebra and $S U(1,1)$ algebra}

Now, we may attempt to solve the Hamiltonian of the 1D multispecies model (12). Motivated by the successful operator approach to single-species model (4) through $S_{N}$-extended Heisenberg algebra, one can try to apply the same techniques to multispecies Hamiltonian. We shall discuss some obstacles to this approach, first recognized in [21].

Following the similar procedure as in Section 2, we define a slightly generalized covariant derivatives $(i=1,2, \ldots, N)$

$$
D_{i}=\partial_{i}+\sum_{j \neq i} \frac{\nu_{i j}}{\left(x_{i}-x_{j}\right)}\left(1-K_{i j}\right)
$$

such that

$$
\left[D_{i}, x_{j}\right]=\delta_{i j}\left(1+\sum_{k=1}^{N} \nu_{i k} K_{i k}\right)-\nu_{i j} K_{i j} .
$$

Without losing generality, and in order to simplify calculations, we set all masses equal to one. Contrary to the previous case the commutator $\left[D_{i}, D_{j}\right]$ does not vanish in general, except on the 
totally symmetric states. In this particular case, the Hamiltonian (13) reads $\tilde{H}=\frac{1}{2} \sum_{i}\left\{a_{i}, a_{i}^{\dagger}\right\}$, where creation and annihilation operators are defined as in (6) (with covariant derivatives (5) replaced by (15)) and their algebra, restricted to the totally symmetric subspace, is

$$
\left[a_{i}, a_{j}^{\dagger}\right]=\delta_{i j}\left(1+\sum_{k=1}^{N} \nu_{i k}\right)-\nu_{i j}, \quad\left[a_{i}^{\dagger}, a_{j}^{\dagger}\right]=\left[a_{i}, a_{j}\right]=0
$$

The vacuum state $(14),|\tilde{0}\rangle=\Delta^{-1}|0\rangle$, is annihilated by $a_{i}$. On this vacuum, we might try to build the towers of excited states. It can be checked that the center of mass excitations, $\mathcal{B}_{1}^{\dagger n}|\tilde{0}\rangle=\left(\sum_{i} a_{i}^{\dagger}\right)^{n}|\tilde{0}\rangle$, are indeed eigenstates of $\tilde{H}$, with eigenenergies $E=E_{0}+n$. The same is true for the Calogero-like state $\mathcal{B}_{2}^{\dagger}|\tilde{0}\rangle=\left(\sum_{i} a_{i}^{\dagger 2}\right)|\tilde{0}\rangle$. Its eigenenergy is $E=E_{0}+2$. However, this procedure breaks down on the next Calogero-like state, namely $\mathcal{B}_{3}^{\dagger}|\tilde{0}\rangle=\left(\sum_{i} a_{i}^{\dagger 3}\right)|\tilde{0}\rangle$. Calculation shows that

$$
\tilde{H}\left(\mathcal{B}_{3}^{\dagger}|\tilde{0}\rangle\right)=\left(E_{0}+3\right)\left(\mathcal{B}_{3}^{\dagger}|\tilde{0}\rangle\right)+\text { const } \cdot \sum_{j \neq i} \frac{\nu_{i j}}{\left(x_{i}-x_{j}\right)}\left(\sum_{l \neq i} \nu_{i l}-\sum_{l \neq j} \nu_{j l}\right)|\tilde{0}\rangle,
$$

i.e. this state is an eigenstate of $\tilde{H}$ only if the all coupling constants $\nu_{i j}$ are equal.

The above result is not so surprising. The particles are no more identical and restriction to totally symmetric space is a too strong requirement. Most of the eigenstates do not have a symmetrical form. Nevertheless, this algebraic approach still reproduces correctly some of the excited states. There is a claim in the literature [35], based on numerical and perturbative analysis of the 3-particle model, that there is no algebra of raising and lowering operators for the multispecies model which gives its complete spectrum. At the moment, we cannot prove or disproves this claim.

As was shown in [15], the Hamiltonian (13) still possesses hidden SU(1,1) structure and one can hope that the additional set of excited states can be determined using an algebraic approach based on $\mathrm{SU}(1,1)$ algebra. Let us define operators

$$
\begin{aligned}
& T_{-}=-\frac{1}{2} \sum_{i} \frac{1}{m_{i}} \frac{\partial^{2}}{\partial x_{i}^{2}}-\frac{1}{2} \sum_{i \neq j} \frac{\nu_{i j}}{\left(x_{i}-x_{j}\right)}\left(\frac{1}{m_{i}} \frac{\partial}{\partial x_{i}}-\frac{1}{m_{j}} \frac{\partial}{\partial x_{j}}\right), \\
& T_{+}=\frac{1}{2} \sum_{i} m_{i} x_{i}^{2} \quad T_{0}=\frac{1}{2}\left(\sum_{i} x_{i} \frac{\partial}{\partial x_{i}}+E_{0}\right),
\end{aligned}
$$

which satisfy $\mathrm{SU}(1,1)$ commutation relations $\left[T_{-}, T_{+}\right]=2 T_{0},\left[T_{0}, T_{ \pm}\right]= \pm T_{ \pm}$. It is possible to construct new set of ladder operators, out of $\mathrm{SU}(1,1)$ generators ( $X$ denotes center-of-mass coordinate and $M$ is total mass of particles)

$$
A_{1}^{ \pm}=\frac{1}{\sqrt{2}}\left(\sqrt{M} X \mp \frac{1}{\sqrt{M}} \frac{\partial}{\partial X}\right), \quad A_{2}^{ \pm}=\frac{1}{2}\left(T_{+}+T_{-}\right) \mp T_{0}
$$

with algebra

$$
\begin{array}{lcc}
\tilde{H}=\left[A_{2}^{-}, A_{2}^{+}\right], & {\left[\tilde{H}, A_{1}^{ \pm}\right]= \pm A_{1}^{ \pm},} & {\left[\tilde{H}, A_{2}^{ \pm}\right]= \pm 2 A_{2}^{ \pm},} \\
{\left[A_{1}^{-}, A_{1}^{+}\right]=1,} & {\left[A_{1}^{-}, A_{2}^{+}\right]=A_{1}^{+},} & {\left[A_{1}^{-}, A_{2}^{-}\right]=\left[A_{1}^{+}, A_{2}^{+}\right]=0 .}
\end{array}
$$

The infinite tower of Fock space-states are built on the vacuum $|\tilde{0}\rangle$ as $\left(n_{1}, n_{2}=0,1, \ldots\right)$

$$
\left|n_{1}, n_{2}\right\rangle \propto A_{1}^{+n_{1}} A_{2}^{+n_{2}}|\tilde{0}\rangle .
$$

Hamiltonian $\tilde{H}$ is diagonal in this basis, i.e.

$$
\tilde{H}\left|n_{1}, n_{2}\right\rangle=\left(E_{0}+\left(n_{1}+2 n_{2}\right)\right)\left|n_{1}, n_{2}\right\rangle .
$$


The energy spectrum is linear in quantum numbers and highly degenerate. It can be shown that the dynamical symmetry responsible for this degeneracy is $\mathrm{SU}(2)$ [15].

If we further define an operator $B_{2}^{ \pm}=A_{2}^{ \pm}-\frac{1}{2} A_{1}^{ \pm 2}$, then we can split the Hamiltonian $\tilde{H}$ in the two parts:

$$
\begin{aligned}
& \tilde{H}_{C M}=\frac{1}{2}\left\{A_{1}^{-}, A_{1}^{+}\right\}_{+}, \quad \tilde{H}_{R}=\left[B_{2}^{-}, B_{2}^{+}\right], \quad\left[\tilde{H}_{C M}, A_{1}^{ \pm}\right]= \pm A_{1}^{ \pm}, \quad\left[\tilde{H}_{R}, B_{2}^{ \pm}\right]= \pm 2 B_{2}^{ \pm}, \\
& \tilde{H}_{C M}|\tilde{0}\rangle_{C M}=\frac{1}{2}|\tilde{0}\rangle_{C M}, \quad \tilde{H}_{R}|\tilde{0}\rangle_{R}=\left(\frac{N-1}{2}+\frac{1}{2} \sum_{i \neq j} \nu_{i j}\right)|\tilde{0}\rangle_{R} .
\end{aligned}
$$

It is clear now that the spectrum (16) consists of center-of-mass mode (CM) and the mode that describes relative motion $(\mathrm{R})$. Furthermore, it is easy to detect the critical point in the Fock space, i.e. the forbidden range of coupling constants $\nu_{i j}$, by inspecting the zero-norm states

$$
\| B_{2}^{+}|\tilde{0}\rangle_{R} \|=0, \quad{ }_{R}\left\langle\tilde{0}\left|\tilde{H}_{R}\right| \tilde{0}\right\rangle_{R}=E_{0 R}=0
$$

It turns out that the critical point is defined by

$$
\sum_{i \neq j} \nu_{i j}=-(N-1)
$$

and tends to the result $\nu=-\frac{1}{N}$ in the limit of identical particles. For $\nu_{i j}$ negative but greater than the critical value (17), the wave functions of the Hamiltonian (13) are singular at coincidence points.

We conclude this Section with a few comments on the multispecies model in arbitrary dimensions [17]. Exactly in the same way as was done in the one-dimensional case, we can find an infinite tower of exact eigenstates for $D$-dimensional multispecies model. These eigenstates are given by $D$-dimensional generalizations of the operators $\left\{A_{1}^{ \pm}, A_{2}^{ \pm}, B_{2}^{ \pm}\right\}$and describe the part of the complete spectrum which corresponds to center-of-mass states and global dilatation states. The $D$-dimensional analogue of the critical point (17) is simply $\sum_{i \neq j} \nu_{i j}=-(N-1) D$.

\section{Conclusion}

In this brief review we described some oscillator aspects of the single-species and multispecies Calogero models in one dimension. We have shown how both type of models could be obtained from the most general (matrix) oscillator Hamiltonian by a suitable "reduction" procedure. We focused on the multispecies variant and tried to solve it. Motivated by the successful operator solution of the single-species Calogero model (based on the $S_{N}$-extended Heisenberg algebra), we applied essentially the same technique to its multispecies counterpart. It turned out that we managed to reproduce only the first two Calogero-like modes. We tried to evade this obstacle by considering underlying SU(1,1) structure of the multispecies Hamiltonian. We defined a new set of creation and annihilation operators using the generators of $\mathrm{SU}(1,1)$. Acting on the ground state, we were able to find algebraically a class of the exact eigenstates and eigenenergies, corresponding to the relative motion and motion of the center of mass. This, SU(1,1)-based, approach can be easy generalized to the more sophisticated models, like multispecies Calogero model in $D$ dimensions [17] or conformal and PT-invariant Hamiltonians described in [36]. However, the problem of finding other exact eigenstates of the multispecies Calogero Hamiltonian(s) still remains open. Nevertheless, we hope that our analysis sheds some light on such kind of models. 


\section{Acknowledgments}

One of the authors (M.M.) would like to thank organizers of the Sixth International Conference "Symmetry in Nonlinear Mathematical Physics" (June 20-26, 2005, Kyiv) for their invitation and warm hospitality. The present paper is a written version of the talk delivered by M.M. at this conference. It is supported by Ministry of Science, Education and Sport of the Republic of Croatia (contracts Nos. 0119261 and 0098003).

[1] Mattis D.C., The many-body problem: 70 years of exactly solved quantum many-body problems, Singapore, World Scientific, 1993.

[2] Sutherland B., Beautiful models, Singapore, World Scientific, 2004.

[3] Calogero F., Ground state of one-dimensional N-body system, J. Math. Phys., 1969, V.10, 2197-2200.

[4] Calogero F., Solution of the one-dimensional $N$-body problems with quadratic and/or inversly quadratic pair potentials, J. Math. Phys., 1971, V.12, 419-436.

[5] Van Dijen J.F., Vinet L. (Editors), Calogero-Moser-Sutherland models, CRM Series in Mathematical Physics, Vol. 25, Berlin, Springer, 2000.

[6] Kawakami N., Critical properties of quantum many-body systems with $1 / r^{2}$ interaction, cond-mat/9402011.

[7] Gibbons G.W., Townsend P.K., Black holes and Calogero models, Phys. Lett. B, 1999, V.454, 187-192, hep-th/9812034.

[8] Sutherland B., Quantum many-body problem in one dimension: ground state, J. Math. Phys., 1971, V.12, 246-250.

[9] Gomis J., Kapustin A., Two-dimensional unoriented strings and matrix models, JHEP, 2004, V.0406, 002, 38 pages, hep-th/0310195.

[10] Gurappa N., Panigrahi P.P., Equivalence of the Calogero-Sutherland model to free harmonic oscillators, Phys. Rev. B, 1999, V.59, R2490-R2493, cond-mat/9710035.

[11] Ezung M., Gurappa N., Khare A., Panigrahi P.K., Quantum many-body systems with nearest and next-tonearest neighbor long-range interaction, Phys. Rev. B, 2005, V.71, 125121, 8 pages, cond-mat/0007005.

[12] Sutherland B., Exact results for a quantum many-body problem in one dimension II, Phys. Rev. A, 1972, V.5, 1372-1376.

[13] Hallnas M., Langmann E., Explicit formulas for the eigenfunctios of the $N$-body Calogero model, mathph/0511040.

[14] Brink L., Hansson T.H., Vasiliev M.A., Explicit solution to the N-body Calogero problem, Phys. Lett. B, 1992, V.286, 109-111, hep-th/9206049.

[15] Meljanac S., Mileković M., Samsarov A., A multispecies Calogero model, Phys. Lett. B, 2003, V.573, 202208, hep-th/0307126.

[16] Meljanac S., Mileković M., Samsarov A., Stojić M., Interacting families of Calogero-type particles and SU(1,1) algebra, Modern Phys. Lett. B, 2004, V.18, 603-612, hep-th/0405132.

[17] Meljanac S., Mileković M., Samsarov A., Generalized Calogero model in arbitrary dimensions, Phys. Lett. B, 2004, V.594, 241-246, hep-th/0405131.

[18] Haldane F.D.M., "Fractional statistics" in arbitrary dimensions: a generalization of the Pauli principle, Phys. Rev. Lett., 1991, V.67, 937-940.

[19] Bernard D., Wu Y.-S., A note on statistical interactions and the thermodynamical Bethe ansatz, in New Developments of Integrable Systems and Long-Ranged interaction Models, Editors M.-L. Ge and Y.-S. Wu, Nankai Lectures on Mathematical Physics, 1995, 10-21, cond-mat/9404025.

[20] Murthy M.V.N., Shankar R., Thermodynamics of a one-dimensional ideal gas with fractional exclusion statistics, Phys. Rev. Lett., 1994, V.73, 3331-3334, cond-mat/9404096.

[21] Furtlehner C., Ouvry S., Calogero model for distinguishable particles, Modern Phys. Lett. A, 1995, V.9, 503-509, hep-th/9407107.

[22] Sen D., A multispecies Calogero-Sutherland model, Nucl. Phys. B, 1996, V.479, 554-574.

[23] Mashkevic S., Towards a quantum-mechanical model for multispecies exclusion statistics, Phys. Lett. A, 1997, V.233, 30-36.

[24] Guhr T., Kohler H., Supersymmetry and models for two kinds of interacting particles, Phys. Rev. E, 2005, V.71, 045102, 4 pages, math-ph/0408033. 
[25] Meljanac S., Samsarov A., Matrix oscillator and Calogero-type models, Phys. Lett. B, 2004, V.600, 179-184, hep-th/0408241.

[26] Bardek V., Jonke L., Meljanac S., Mileković M., Calogero model, deformed oscillators and the collapse, Phys. Lett. B, 2002, V.531, 311-315, hep-th/0107053.

[27] Meljanac S., Mileković M., Stojić M., Permutation invariant algebras, a Fock space realization and the Calogero model, Eur. Phys. J. C Part. Fields, 2002, V.24, 331-343, math-ph/0201061.

[28] Polychronakos A.P., Exchange operator formalism for integrable system of particles, Phys. Rev. Lett., 1992, V.69, 703-705, hep-th/9202057.

[29] Jonke L., Meljanac S., Bosonic realization of algebras in the Calogero model, Phys. Lett. B, 2002, V.256, 149-156, hep-th/0106135.

[30] Jonke L., Meljanac S., Dynamical symmetry algebra of the Calogero model, Phys. Lett. B, 2001, V.511, 276-284, hep-th/0105043.

[31] Meljanac S., Mileković M., A unified view of multimode algebras with Fock-like representations, Internat. J. Modern Phys. A, 1996, V.11, 1391-1412.

[32] Gorsky A., Nekrasov N., Hamiltonian systems of Calogero type and two dimensional Yang-Mills theory, Nucl. Phys. B, 1994, V.414, 213-238, hep-th/9304047.

[33] Calogero F., Marchioro C., Exact bound states of some $N$-body systems with two- and three-body forces, J. Math. Phys., 1973, V.14, 182-184.

[34] Forrester P.J., Some multidimensional integrals related to many-body systems with the $1 / r^{2}$ potential, J. Phys. A: Math. Gen., 1992, V.25, L607-L614.

[35] Dasniers de Veigy A., On the solution of the Calogero model distinguishable particles in the domain of intermediate statistics, Nucl. Phys. B, 1997, V.483, 580-600.

[36] Meljanac S., Samsarov A., Universal properties of conformal quantum many-body systems, Phys. Lett. B, 2005, V.613, 221-225, hep-th/0503174. 